\title{
Agroindustria de la caña de azúcar: desafíos para el sector agrícola
}

Sugarcane agroindustry: challenges for the agricultural sector

Jessica Liliana Castillo Torres ${ }^{a}$, Diana Xóchitl González Gómez ${ }^{b}$, Jozelin Maria Soto Alarcón ${ }^{c}$

\begin{abstract}
:
The sugar cane agroindustry is relevant in the agricultural production of Mexico. In 2016, this crop represented 7.3\% of the subsector's GDP and generated around 440 thousand jobs. In addition, the federal government has promoted its production through CONADESUCA, to coordinate actions that promote competitiveness and innovation for its development. However, the contribution to production in the state of Hidalgo in 2016 was only $0.47 \%$, and shows a decreasing trend from 2005 to 2016 . Due to the above, the present research identifies areas of opportunity for the growth and diversification of the agroindustry in the State of Hidalgo, for that the SAGARPA indicates that sugar cane is used to generate various products and subproducts, as sugar, fertilizer, animal feed, alcohol. Based on data from the SIACON, it is found that sugar cane is produced in twenty municipalities of the State, located in the northern regions, in two periods 2003-2006 and 2014-2016. The use of this crop in Hidalgo is only for the production of piloncillo.
\end{abstract}

Keywords:

Q1: Agriculture, R11: Regional Economic Activity: Growth, Development, Environmental Issues, and Changes, O13: Agriculture; Natural Resources; Energy; Environment; Other Primary Products.

\section{Resumen:}

La agroindustria de la caña de azúcar es relevante en la producción agropecuaria de México. En 2016, este cultivo representó el 7.3\% del PIB del subsector y generó alrededor de 440 mil empleos. Además, el gobierno federal ha impulsado su producción a través del CONADESUCA, para coordinar acciones que fomenten la competitividad e innovación para su desarrollo. Sin embargo, la contribución a la producción en el estado de Hidalgo en el 2016 fue de apenas el $0.47 \%$, y muestra una tendencia decreciente del 2005 al 2016. Por lo anterior, el presente trabajo de investigación identifica las áreas de oportunidad para el crecimiento y diversificación de la agroindustria en la entidad hidalguense, ya que la SAGARPA señala que la caña de azúcar se utiliza para generar diversos productos y subproductos, como azúcar, abono, alimento para animales, alcohol. Con base en datos del SIACON, se encuentra que la caña de azúcar se produce en veinte municipios del Estado, ubicados en las regiones del norte, en dos periodos 2003-2006 y 20142016. El uso de este cultivo en Hidalgo es únicamente para la producción de piloncillo.

\section{Palabras Clave:}

Q1: Agricultura, R11: Actividad económica regional: crecimiento, desarrollo, problemas ambientales y cambios, O13: Agricultura; Recursos naturales; Energía; Ambiente; Otros productos primarios.

\section{Introducción}

La siembra y cosecha de caña azúcar en México es un cultivo que ha mostrado una tendencia productiva creciente de 1961 a 2015, a pesar de la crisis financiera que presenta el sector desde 1988. La política económica hacia el sector cañero ha pasado de la intervención directa del Estado hasta 1991 a la desregulación, promoción de la competitividad y la apertura comercial. 1 La industria de caña mexicana se enfrenta a un mercado internacional que comercia alrededor de 160 millones de toneladas tanto de caña de azúcar como de remolacha, los principales productores son Brasil, India, la Unión Europea y México ocupó el lugar número siete de 2014 a 2016. A partir del ciclo agrícola del 2015-2016 el mercado de azúcar se enfrenta a una demanda creciente de los consumidores. 2 Además, la agroindustria azucarera se ha diversificado en el tiempo, de la producción de

\footnotetext{
a Universidad Autónoma del Estado de Hidalgo, Estudiante de la Licenciatura en Economía del Instituto de Ciencias Económico Administrativas, Email: je_li_96@hotmail.com

${ }^{\mathrm{b}}$ Autor de Correspondencia. Universidad Autónoma del Estado de Hidalgo, Instituto de Ciencias Económico Administrativas, ORCID: 00000003-3062-9961, Email: dianax@uaeh.edu.mx
} 
sacarosa, melazas y bagazo se derivan al menos 15 productos con alto valor en el mercado. 3

Debido a la importancia productiva de la agroindustria azucarera, este estudio tiene como objetivo analizar las exportaciones e importaciones de la caña de azúcar e identificar el papel de México en el mercado mundial y por otro lado estudiar el nivel de la producción municipal en el estado de Hidalgo de 2003 a 2005 y de 2014 a 2016. En la tercera sección se analiza la producción nacional y el procesamiento de la caña de azúcar, la política pública implementada desde 2008 para promover el desarrollo sustentable de la agroindustria y los mecanismos para aliarse con otros programas públicos. En la cuarta sección se presentan el estudio municipal de la producción de caña de azúcar en el estado de Hidalgo a nivel municipal de 2003 a 2005 y de 2014 a 2016. Finalmente, en la sección cinco se presentan los hallazgos.

\section{Agroindustria: definición y alcances}

La agroindustria se refiere al conjunto de actividades que se realizan una vez que se ha cosechado, como son la transformación, preservación y preparación de la producción agrícola para el consumo intermedio o final. Es una fuente de creación de ingresos y de empleo en los países en desarrollo. 4

La perspectiva del Banco Mundial sobre la agricultura es que no solo sea vista como una actividad económica del sector primario, sino también como una forma de reducir la pobreza, de promover la gestión ambiental, la inocuidad alimentaria y el bienestar de la población. 5

Desde finales del siglo $\mathrm{XX}$, la agroindustria azucarera en México ya se veía afectada por el descenso de los precios internacionales del azúcar, la sobreproducción y la creciente incorporación de sustitutos más baratos como es el jarabe de maíz de alta fructuosa, procedente de Estados Unidos. 6 Asimismo, con la entrada del Tratado de Libre Comercio de América del Norte (TLCAN), se eliminó el arancel a la importación de maíz amarillo de Estados Unidos, y con ello, se ha incrementado la producción interna de fructosa. 6 pág. 244.

\section{Caña de azúcar}

La caña de azúcar es un cultivo de gran importancia en México y en los más de 130 países que se cultiva a nivel mundial. Su valor económico se basa en tres atributos: a) es altamente productiva, b) es muy eficiente en el uso de insumos y recursos productivos, y c) puede ser procesada de manera local y generar productos con valor agregado, tales como sacarosa, melaza, etanol y energía. 7 pág. 8.

El crecimiento de la producción cañera en México se debió al incremento en la superficie cultivada durante 2006 y 2011, más que al rendimiento. La reconversión de zonas maiceras a cañeras y la tecnificación por riego, son algunos factores que explican el aumento de la superficie cultivada y de un incremento en el rendimiento, respectivamente. 8 Después de 2011 se registró una caída en los precios, afectando la producción.

En el Diario Oficial de la Federación se señala que:

"La dinámica de la producción de azúcar depende de factores diversos. El azúcar se produce a partir de la caña de azúcar y de la remolacha azucarera (principalmente en la Unión Europea y, en menor medida, en Estados Unidos). Compite por superficie sembrada con otros cultivos, además, la caña de azúcar se emplea también para la producción de etanol. Si atendemos a su estructura de costos, la producción de azúcar es intensiva en capital, por lo cual mantiene costos fijos elevados. Otro factor de gran relevancia, sin duda, son las variaciones en las condiciones climáticas que afectan a las zonas productoras de caña de azúcar. En ese contexto, la producción de azúcar se verá afectada por cambios en otros mercados agrícolas, por las decisiones de producción de etanol a partir de la caña de azúcar (particularmente de Brasil), de la rentabilidad asociada a la evolución de los precios del azúcar y de sus sustitutos, así como de las condiciones climáticas que prevalezcan en cada ciclo productivo." 9 pág. 4.

Los residuos de la caña de azúcar en su estado natural constituyen el $28 \%$ aproximadamente. 10 Es decir, el $19.74 \%$ es paja que se obtiene del secado de las hojas y el $8.44 \%$ es el cogollo, parte verde de la planta que queda en el campo. Estos constituyen una fuente de energía renovable. Los autores señalan que algunos países productores de este cultivo, se desarrollan proyectos para generar combustible vegetal a partir de la paja residual. Se añade valor agregado y con su uso se evita un fuerte impacto ambiental provocado por la quema de la paja. La paja picada también se siembra con maíz, trigo, avena o sorgo, para producir forraje animal. Además, puede tener otras aplicaciones en productos artesanales o ser utilizada como fertilizante para suelos.

De la cosecha y procesamiento de la caña de azúcar se pueden obtener ocho productos y subproductos como son: sacarosa, etanol, residuos de cosecha, bagazo, melazas, cachaza, vinazas y cenizas de calderas. Sin embargo, los estudios señalan que no ha habido un modelo nacional de diversificación en la industria cañera mexicana. 11

\section{Mercado internacional}

La actividad agropecuaria en México se ha posicionado en el mercado internacional desde hace algunas décadas. El cuadro 1 muestra el listado de los veinte commodities de exportación de mayor importancia para la actividad 
primaria en 2013, último año del que se dispone de información de FAOSTAT.

Cuadro 1. Principales productos primarios de exportación de México en 2013.

\begin{tabular}{|c|c|c|}
\hline Posición & Producto & $\begin{array}{l}\text { Exportación (valor } \\
\text { en dólares US) }\end{array}$ \\
\hline 1 & Cerveza de cebada & $2,211,205,000$ \\
\hline 2 & Tomates, frescos & $1,835,175,000$ \\
\hline 3 & Bebidas, alcohólicas destiladas & $1,148,115,000$ \\
\hline 4 & Aguacates & $1,086,935,000$ \\
\hline 5 & $\begin{array}{l}\text { Chiles, pimientos picantes, pimientos } \\
\text { (verdes) }\end{array}$ & $867,659,000$ \\
\hline 6 & Azúcar, refinada & $787,845,000$ \\
\hline 7 & $\begin{array}{l}\text { Alimento, Preparados para consumo } \\
\text { humano nep }\end{array}$ & $750,829,000$ \\
\hline 8 & Pastelería & $730,360,000$ \\
\hline 9 & Chocolate, productos nep & $598,437,000$ \\
\hline 10 & Azúcar, dulces, confitería & $565,549,000$ \\
\hline 11 & Azuc Cent Nr & $516,547,000$ \\
\hline 12 & Fruta, preparada, nep & $483,239,000$ \\
\hline 13 & Café, verde & $466,247,000$ \\
\hline 14 & Carne, vacuno deshuesada & $455,571,000$ \\
\hline 15 & Carne, cerdo preparada & $444,250,000$ \\
\hline 16 & Pepinos, pepinillos & $440,647,000$ \\
\hline 17 & Calabazas, zapayo, calabaza confitera & $402,949,000$ \\
\hline 18 & Hortalizas, congeladas & $370,910,000$ \\
\hline 19 & Cebollas, secas & $363,721,000$ \\
\hline 20 & Alimentos infantiles & $353,822,000$ \\
\hline
\end{tabular}

\section{Producción mundial de caña de azúcar}

El cultivo de la caña de azúcar se produce en diversas regiones del mundo. Brasil produce el $49 \%$ de la producción total, India el $21 \%$, China $8 \%$, Tailandia $6 \%$, Pakistán 4\% al igual que México (4\%). Estos países junto con Colombia, Australia y Guatemala integran el top 10 de producción azucarera en el mundo. La economía mexicana ocupa el sexto lugar (gráfico 1).

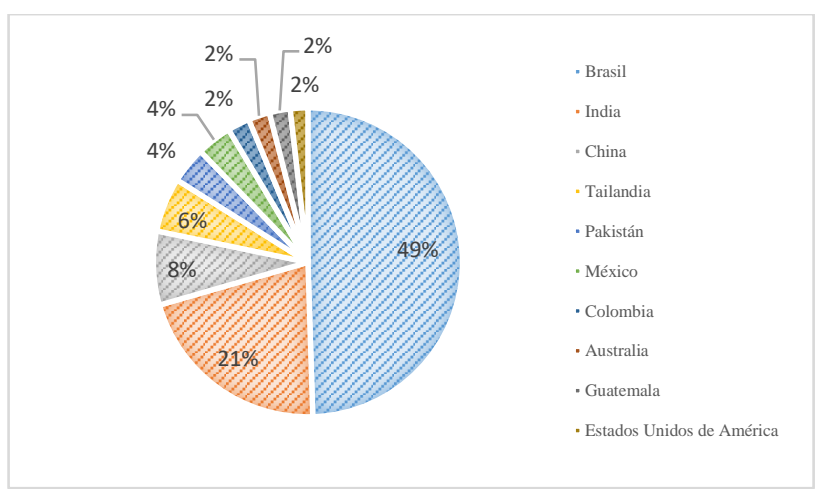

Gráfico 1. Participación porcentual en la producción mundial de caña de azúcar por país en 2016

\section{Exportación de azúcar refinada}

A partir de los años noventa México empezó a posicionarse en el top 20 de la exportación mundial de azúcar refinada. De acuerdo al gráfico 2, en los años 1997 y 1999 se ubicó en el lugar 15, sin embargo, en el año 2013 logró la cuarta posición.

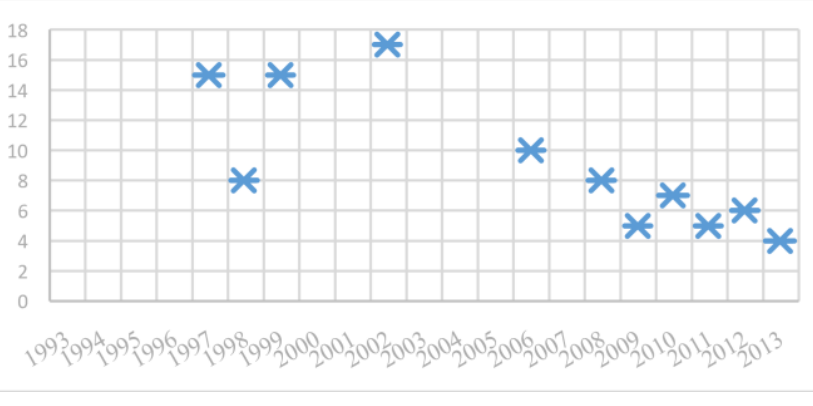

Gráfico 2. Posición mundial en el valor de exportación de azúcar refinada de México

El gráfico 3 muestra que el valor de las exportaciones de azúcar refinada de México tiene cortes estructurales en la serie temporal de 1970 a 2013. Debido a que los valores están a precios de 2010 , los cambios en la serie se deben únicamente a las variaciones, en términos reales, del valor de las exportaciones. Es decir, se observa una tendencia creciente de la serie en este periodo. En el año 2011 es cuanto alcanza el valor máximo.

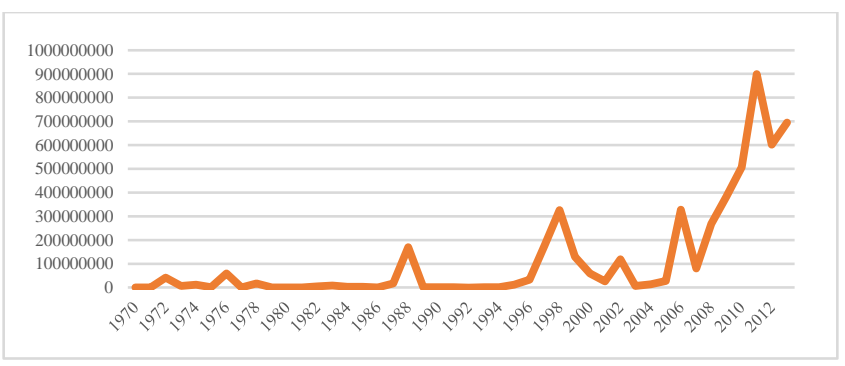

Gráfico 3. Valor de las exportaciones de azúcar refinada en México, a precios de 2010

\section{Importación de caña de azúcar}

Las estadísticas de FAOSTAT muestran que, en 2013, último año disponible de información, Italia es el país que más importa azúcar refinada por el valor de las importaciones en dólares. Estados Unidos se ubica en el segundo lugar, y también es el principal destino de las exportaciones azucareras mexicanas. 


\begin{tabular}{|c|l|c|}
\hline Posición & \multicolumn{1}{|c|}{ País } & $\begin{array}{c}\text { Valor de importaciones de } \\
\text { azúcar refinada }\end{array}$ \\
\hline 1 & Italia & $1,116,810.00$ \\
\hline 2 & $\begin{array}{l}\text { Estados Unidos } \\
\text { de América }\end{array}$ & $864,228.00$ \\
\hline 3 & $\begin{array}{l}\text { Emiratos Árabes } \\
\text { Unidos }\end{array}$ & $608,391.00$ \\
\hline 4 & Alemania & $548,256.00$ \\
\hline 5 & España & $481,732.00$ \\
\hline 6 & Sudán & $445,640.00$ \\
\hline 7 & Bélgica & $427,470.00$ \\
\hline 8 & Arabia Saudita & $394,625.00$ \\
\hline 9 & Irak & $362,022.00$ \\
\hline 10 & Yemen & $332,484.00$ \\
\hline
\end{tabular}

Cuadro 2. Principales países importadores de azúcar refinada en 2013, en miles de dólares internacionales

\section{Mercado nacional}

La caña de azúcar constituye la agroindustria más antigua del país y cuenta con una ubicación geográfica estratégica. Este cultivo se produce durante la zafra (es el tiempo que dura el proceso mediante el cual se cosecha la caña y se fabrica el azúcar), que comienza en noviembre y finaliza en julio. Su producción es una importante fuente de empleos debido a que todo el proceso de fabricación de los derivados requiere de mucha mano de obra, por lo que este cultivo genera alrededor de 440 mil empleos.

En 2016 se cultivaba en 22 estados de la República Mexicana, entre los que destacan Veracruz, que aporta el $41.6 \%$ a la producción nacional, San Luis Potosí con un $11.2 \%$, y Jalisco el $9.6 \%$. El principal uso de la caña de azúcar en el país es para la fabricación de azúcar, aunque también se utiliza en la fabricación de papel, abonos, alimento para animales y para la fabricación de alcohol. Es uno de los cultivos más consumidos por la población mexicana, según SAGARPA (Secretaría de Agricultura, Ganadería, Desarrollo Rural, Pesca y Alimentación) su consumo representa el $5 \%$ del consumo total de alimentos y bebidas.

\section{Regiones Estratégicas}

Para el desarrollo productivo nacional y regional de la caña de azúcar se han establecido 17 regiones potenciales bajo las estrategias de "maximizar" e "incentivar" la producción. De acuerdo a SAGARPA, la primera consiste en implementar una campaña nacional para la eficiencia en el manejo de agua en los ingenios azucareros; en impulsar la tecnificación del riego; en fortalecer las bases de información que contienen los precios internacionales del producto y la toma de decisiones de su producción; en reforzar la vinculación con el sector productivo para la investigación, la transferencia de tecnología y las innovaciones en campo y fábrica para incrementar su productividad; en promover la diversificación de los procesos agroindustriales del cultivo; en estandarizar el uso eficiente de fertilizantes; en mejorar el financiamiento para que sea oportuno y competitivo; y en adquirir herramientas y mecanismos para la administración de riesgos, y la segunda hace referencia a incentivar la conversión de temporal a riego y en impulsar la compactación de hectáreas para producir en unidades de 30 a 50 hectáreas.

La SAGARPA establece que los motores de planeación para lograr dicho desarrollo y poder llevar a cabo las estrategias son la creación de políticas agrícolas eficientes, la existencia de financiamientos para la productividad, la educación agrícola, la existencia de información del sector agrícola, la productividad con enfoque de rentabilidad, la sustentabilidad en la producción agrícola, la creación de logística y mercados, la aplicación de tecnología al campo, y como factores fundamentales y de vital importancia la investigación, la innovación y el desarrollo tecnológico.

En el cuadro 3 se enlistan las 17 regiones según SAPARPA, los estados que las conforman y sus principales objetivos para el periodo 2017-2030.

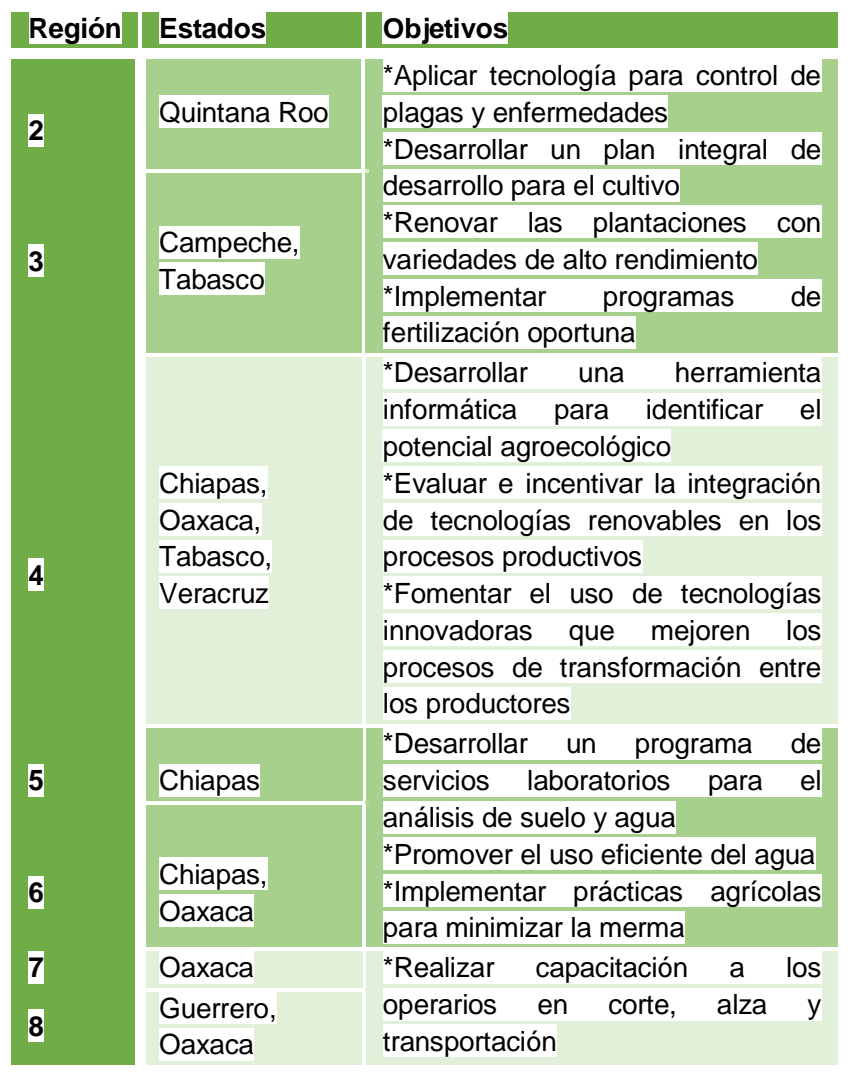




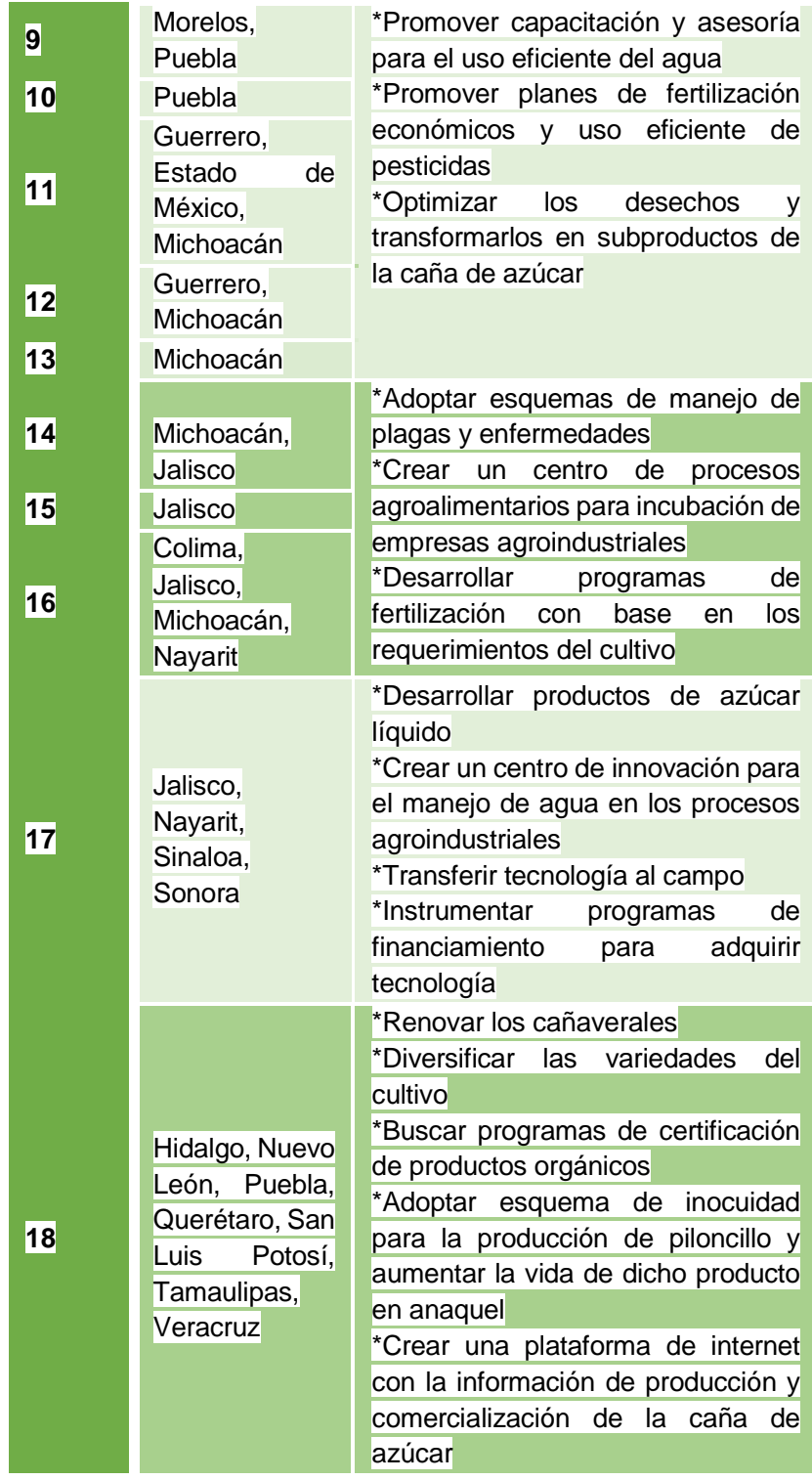

Cuadro 3. Regiones estratégicas para el desarrollo productivo de caña de azúcar

\section{Ingenios azucareros}

En México, durante el periodo 2015-2016 se tenían registrados 51 ingenios azucareros, distribuidos en siete regiones cañeras, en el CONADESUCA (Comité Nacional para el Desarrollo Sustentable de la Caña de Azúcar) dependiente de la SAGARPA.

Según la SAGARPA, los ingenios azucareros son el primer paso de la transformación del azúcar. Se conoce como ingenio azucarero a la planta industrial dedicada al procesamiento, transformación e industrialización de la caña de azúcar, sus principales actividades son la producción de azúcar y la refinación de la misma. La primera actividad consiste en nueve pasos, los cuales son corte de la caña; molienda; generación de vapor; calentamiento; clarificación; filtración; evaporación; cristalización; evaporado y secado. La segunda actividad consiste en separación de sólidos; alcalización; clarificación; decoloración; filtración; evaporación; cristalización; centrifugado; y secado por medio de corrientes de aire. Con la refinación se pueden obtener cuatro tipos de azúcar: mascabado, estándar, refinado y blanco.

\begin{tabular}{|c|c|c|}
\hline Región & Entidades & $\begin{array}{l}\text { Número de } \\
\text { ingenios }\end{array}$ \\
\hline tro & $\begin{array}{l}\text { Morelos, Estado de } \\
\text { México, Puebla, Veracruz }\end{array}$ & 5 \\
\hline doba-Golfo & Oaxaca, Veracruz & 10 \\
\hline reste & $\begin{array}{l}\text { Tamaulipas, San Luis } \\
\text { Potosí, Veracruz }\end{array}$ & 8 \\
\hline oeste & Sinaloa, Nayarit & 3 \\
\hline cífico & $\begin{array}{l}\text { Jalisco, Colima, Michoacán } \\
\text { de Ocampo }\end{array}$ & 10 \\
\hline $\begin{array}{l}\text { baloapan- } \\
\text { Ifo }\end{array}$ & Oaxaca, Veracruz & 8 \\
\hline este & $\begin{array}{l}\text { Tabasco, Chiapas, } \\
\text { Campeche, Quintana Roo }\end{array}$ & 7 \\
\hline
\end{tabular}

Cuadro 4. Ingenios azucareros en México

\section{Producción de caña de azúcar}

El gráfico 4 muestra la producción de caña de azúcar en México medido en toneladas, y el rendimiento en toneladas por hectárea. Se observa que el volumen de producción ha tenido un comportamiento creciente desde 1960 a la primera década del siglo XXI, en 2013 se incrementó la producción considerablemente, debido al aumento en la superficie sembrada; sin embargo, el rendimiento se ha mantenido constante en los últimos años. 12

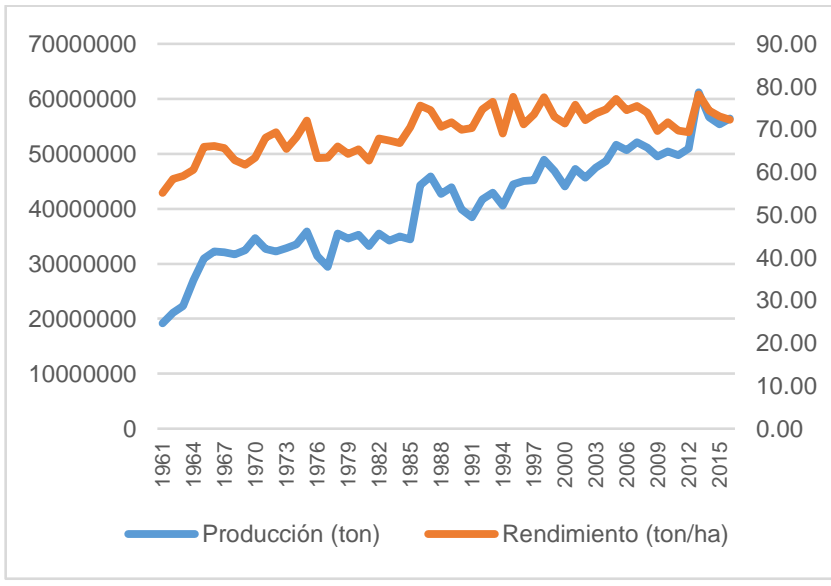

Gráfico 4. Volumen de producción y rendimiento de la caña de azúcar en México 


\section{Programas federales de apoyo a la producción de caña de azúcar}

El CONADESUCA es un organismo mexicano dedicado a coordinar de manera efectiva las acciones de vinculación, promoción, fomento, competitividad e innovación para el desarrollo sustentable de la agroindustria de la caña de azúcar, mediante la planeación y aplicación de políticas públicas que den seguridad a todos los productores de este cultivo en su toma de decisiones. Este comité trabaja conforme los lineamientos del Sistema Integral de Información y el CICTCAÑA (Centro de Investigación Científica y Tecnológica de la Caña de Azúcar que se encarga de orientar, integrar, dirigir y estructurar los trabajos de investigación científica nacional para la competitividad de la agroindustria de la caña de azúcar a nivel nacional). El CONADESUCA en conjunto con la SAGARPA, elaboraron el Programa Nacional de la Caña de Azúcar (PRONAC) 2014-2018, el cual establece como sus principales estrategias reducir los costos de producción; incentivar acciones que incrementen la productividad del sector; promover la diversificación productiva de la caña de azúcar; fomentar el acceso a financiamiento adecuado y oportuno; fomentar prácticas y tecnologías sustentables en los procesos productivos; y fomentar la investigación, desarrollo, innovación y transferencia de tecnología.

Como parte de sus objetivos, la SAGARPA establece que es necesario crear políticas de apoyo para mejorar la producción; aprovechar las ventajas competitivas del sector agropecuario e integrar las actividades del sector a las cadenas productivas de la economía mexicana; todo esto se debe lograr mediante la colaboración de las organizaciones de productores con programas y proyectos de la secretaría (Cuadro 5)

\begin{tabular}{|c|c|c|c|}
\hline Programas & Objetivo & Objetivo Específico & $\begin{array}{l}\text { Población } \\
\text { Objetivo }\end{array}$ \\
\hline $\begin{array}{l}\text { Programa } \\
\text { de fomento } \\
\text { a la } \\
\text { Agricultura }\end{array}$ & $\begin{array}{l}\text { Incrementar la } \\
\text { productividad } \\
\text { agrícola }\end{array}$ & $\begin{array}{l}\text { Incrementar la producción y la } \\
\text { productividad del sector agrícola } \\
\text { incentivando: } \\
\text { - La integración de cadenas } \\
\text { productivas } \\
\text { - } \quad \text { El desarrollo de Agroclúster } \\
\text { - Inversión de capital humano, } \\
\text { físico y tecnológico } \\
\text { - El uso eficiente de energía } \\
\text { - El uso sustentable de los } \\
\text { recursos naturales }\end{array}$ & $\begin{array}{l}\text { Personas y } \\
\text { morales y } \\
\text { organizacione } \\
\text { s que } \\
\text { desempeñen } \\
\text { actividades } \\
\text { primarias, de } \\
\text { transformació } \\
\text { n y } \\
\text { comercializaci } \\
\text { ón }\end{array}$ \\
\hline $\begin{array}{l}\text { Programa } \\
\text { Integral de } \\
\text { Desarrollo } \\
\text { Rural }\end{array}$ & $\begin{array}{l}\text { Reducir la } \\
\text { inseguridad } \\
\text { alimentaria de } \\
\text { la población } \\
\text { en pobreza } \\
\text { extrema de } \\
\text { zonas rurales } \\
\text { marginadas y } \\
\text { periurbanas }\end{array}$ & $\begin{array}{l}\text { Incrementar la producción de } \\
\text { alimentos con incentivos para: } \\
\text { - La adquisición de insumos y } \\
\text { equipamiento productivo } \\
\text { - La construcción de } \\
\text { infraestructura } \\
\text { El aprovechamiento } \\
\text { sustentable de suelo y agua } \\
\text { El desarrollo productivo, de } \\
\text { capacidades y servicios } \\
\text { profesionales de extensión e } \\
\text { innovación rural } \\
\text { El fortalecimiento de las } \\
\text { organizaciones rurales } \\
\text { La creación de esquemas de } \\
\text { aseguramiento para } \\
\text { afectaciones por desastres } \\
\text { naturales }\end{array}$ & $\begin{array}{l}\text { Personas } \\
\text { físicas y } \\
\text { morales que } \\
\text { realizan } \\
\text { actividades } \\
\text { agrícolas, } \\
\text { pecuarias, } \\
\text { acuícolas y } \\
\text { pesqueras en } \\
\text { zonas rurales } \\
\text { y periurbanas }\end{array}$ \\
\hline $\begin{array}{c}\text { Programa } \\
\text { de } \\
\text { productivi } \\
\text { dad y } \\
\text { competitivi } \\
\text { dad }\end{array}$ & $\begin{array}{l}\text { Impulsar la } \\
\text { productividad } \\
\text { y } \\
\text { competitividad } \\
\text { en el sector }\end{array}$ & $\begin{array}{l}\text { Las unidades económicas del Sector } \\
\text { Agroalimentario deben } \\
\text { Invertir en el desarrollo de } \\
\text { capital físico, humano y } \\
\text { tecnológico }\end{array}$ & $\begin{array}{l}\text {-Productores } \\
\text { agropecuarios } \\
\text { y pesqueros } \\
\text {-Personas } \\
\text { físicas y } \\
\text { morales que }\end{array}$ \\
\hline
\end{tabular}

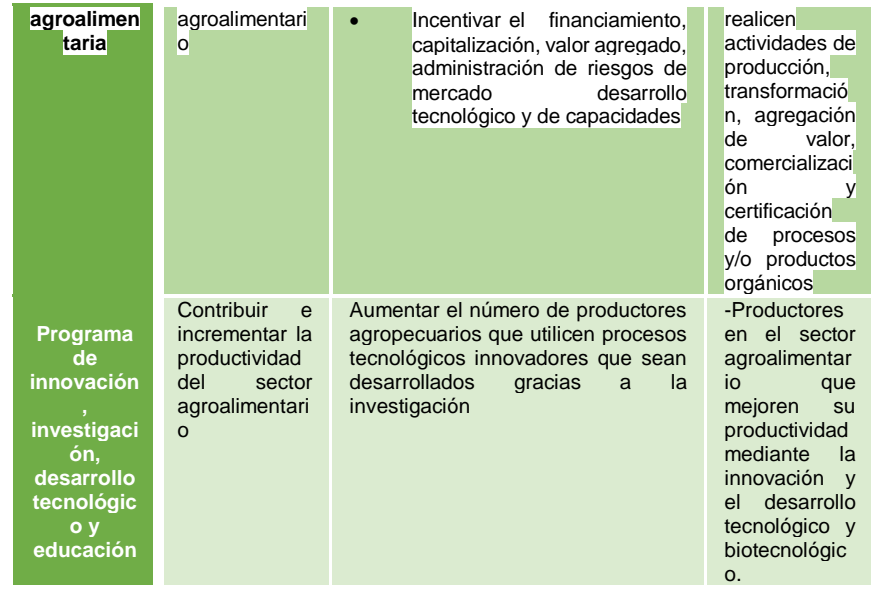

Cuadro 5. Programas de apoyo para la producción de caña de azúcar

\section{Metodología}

Con base en las Estadísticas de la Producción Agrícola del Servicio de Información Agroalimentaria y Pesquera (SIAP) se construyen matrices de porcentajes de participación del valor de la producción de caña de azúcar por municipio del estado de Hidalgo, en los periodos 20032006 y 2014-2016. La información cuantitativa por municipio que proporciona el SIAP inicia a partir del año 2003, además de que es en ese mismo año cuando se liberalizan todos los productos agrícolas conforme a los lineamientos del TLCAN (Tratado de Libre Comercio de América del Norte), por esa razón se considera el primer periodo de estudio 2003-2006. Y el segundo periodo (2014-2016) corresponde a los tres últimos años de que se dispone de información. Se utiliza el deflactor agropecuario de la FAO (Food and Agriculture Organization) para estimar el valor de la producción a precios constantes del año 2010.

\section{Producción en el estado de Hidalgo}

La caña de azúcar se produce en veinte municipios de la entidad hidalguense, ubicados en las regiones Huasteca, Sierra Alta, Sierra Baja y Sierra Otomí-Tepehua. En el año 2003 los municipios que se ubican en las primeras cinco posiciones por el valor de su producción (a precios constantes de 2010) son: Huejutla de Reyes (1\%), Tianguistengo $\left(2^{\circ}\right)$, Yahualica $\left(3^{\circ}\right)$, Tepehuacán de Guerrero (4ํ) y San Bartolo Tutotepec (5ํ). En el año 2016 algunos de estos municipios se mantienen en las primeras posiciones, tales como: Huejutla de Reyes ( $\left.1^{\circ}\right)$, Yahualica $\left(2^{\circ}\right)$, Tepehuacán de Guerrero ( $\left.3^{\circ}\right)$; sin embargo, en el cuarto lugar se ubica San Felipe Orizatlán y Calnali en la sexta posición (cuadro 6). 


\begin{tabular}{|c|c|c|c|c|c|c|c|c|}
\hline \multirow[b]{2}{*}{ Municipio } & \multicolumn{8}{|c|}{ Porcentaje de participación } \\
\hline & $\begin{array}{c}\text { Posición } \\
\text { en el } \\
\text { Estado } \\
(2003)\end{array}$ & 2003 & 2004 & 2005 & 2014 & 2015 & 2016 & $\begin{array}{c}\text { Posición } \\
\text { en el } \\
\text { Estado } \\
(2016)\end{array}$ \\
\hline Atlapexco & 7 & 4.9 & 5.9 & 5.7 & 5.5 & 5.4 & 7.2 & 6 \\
\hline Calnali & 6 & 7.6 & 4.8 & 4.1 & 7.1 & 9.6 & 7.5 & 5 \\
\hline Chapulhuacán & 13 & 1.1 & 1.2 & 1.1 & 0.8 & 0.7 & 0.6 & 19 \\
\hline Huautla & 15 & 0.8 & 1.0 & 1.0 & 1.3 & 1.1 & 1.2 & 16 \\
\hline Huazalingo & 9 & 3.7 & 3.9 & 4.3 & 4.1 & 4.1 & 3.9 & 8 \\
\hline Huehuetla & 11 & 1.6 & 1.7 & 1.4 & 3.2 & 3.6 & 3.6 & 9 \\
\hline $\begin{array}{l}\text { Huejutla de } \\
\text { Reyes }\end{array}$ & 1 & 18.3 & 18.9 & 22.8 & 23.4 & 20.1 & 20.2 & 1 \\
\hline Jaltocán & 17 & 0.7 & 0.8 & 0.8 & 0.8 & 0.8 & 0.8 & 18 \\
\hline Lolotla & 16 & 0.7 & 0.9 & 0.8 & 1.9 & 2.1 & 1.8 & 12 \\
\hline $\begin{array}{l}\text { Molango de } \\
\text { Escamilla }\end{array}$ & 8 & 4.7 & 1.3 & 1.4 & 0.03 & 1.6 & 1.6 & 14 \\
\hline $\begin{array}{l}\text { San Bartolo } \\
\text { Tutotepec }\end{array}$ & 5 & 10.4 & 1.6 & 1.4 & 2.6 & 2.9 & 3.0 & 10 \\
\hline $\begin{array}{l}\text { San Felipe } \\
\text { Orizatlán }\end{array}$ & 12 & 1.4 & 13.4 & 13.5 & 8.4 & 8.9 & 9.2 & 4 \\
\hline $\begin{array}{l}\text { Tenango de } \\
\text { Doria }\end{array}$ & 20 & 0.4 & 0.4 & 0.4 & 0.9 & 0.9 & 0.9 & 17 \\
\hline $\begin{array}{l}\text { Tepehuacán } \\
\text { de Guerrero }\end{array}$ & 4 & 10.7 & 8.1 & 5.8 & 10.3 & 9.0 & 10.3 & 3 \\
\hline Tianguistengo & 2 & 15.5 & 16.4 & 15.8 & 2.9 & 3.0 & 2.9 & 11 \\
\hline Tlanchinol & 19 & 0.6 & 0.6 & 0.5 & 1.3 & 1.5 & 1.4 & 15 \\
\hline Xochiatipan & 10 & 3.6 & 4.2 & 4.2 & 5.3 & 4.9 & 4.4 & 7 \\
\hline Xochicoatlán & 18 & 0.6 & 0.5 & 0.6 & 1.0 & 0.9 & 1.7 & 13 \\
\hline Yahualica & 3 & 11.0 & 12.9 & 13.2 & 17.9 & 17.3 & 16.3 & 2 \\
\hline $\begin{array}{l}\text { Zacualtipán } \\
\text { de Ángeles }\end{array}$ & 14 & 0.8 & 0.5 & 0.4 & 0.3 & 0.3 & 0.4 & 20 \\
\hline
\end{tabular}

Cuadro 6. Porcentaje de participación por municipio en el valor de la producción de caña de azúcar

\section{Resultados}

El municipio que aporta un mayor valor de producción de caña de azúcar al Estado es Huejutla de Reyes durante el periodo de estudio, su aportación en 2003 era del 18.3\% y en 2016 de $20.2 \%$. Algunos otros municipios han disminuido su producción en el total de la entidad, es el caso de Tianguistengo al pasar del segundo al onceavo lugar.

El cultivo de la caña de azúcar en México tiene cuatro usos: a) para la producción de caña de azúcar, b) para forraje, c) para fruta y d) para la producción de piloncillo. En el estado de Hidalgo, todo el cultivo se destina a la producción de piloncillo, de acuerdo a los resultados del análisis de porcentajes de participación de la producción por municipio en la entidad. A diferencia de Guanajuato y Zacatecas que en 2016 se especializaron en el uso del cultivo para la producción de fruta, o bien, Quintana Roo, Sinaloa, Tabasco y Tamaulipas que en el mismo año se especializaron en la producción de caña de azúcar. Existen otros municipios que diversifican el cultivo en todos sus usos, tal es el caso de Nayarit. El estado de Guerrero diversifica el cultivo para forraje, fruta y piloncillo, así como Veracruz cuya producción es para fruta, piloncillo y azúcar.

\section{Conclusiones}

En el comercio internacional de caña de azúcar, México ocupa el sexto lugar en la producción mundial, aportando el $4 \%$ al valor en el año 2016. Sin embargo, es el azúcar refinada la que exporta nuestro país, situándose en el cuarto lugar de los principales países exportadores en el año 2013. El principal destino del cultivo es Estados Unidos, quien se encuentra como el segundo país comprador más importante a nivel mundial.

En el entorno nacional, el gobierno federal ha implementado diversos programas en apoyo a la producción cañera a través de organismos como el CONADESUCA y la creación de las "Regiones Estratégicas" que determinan los objetivos específicos y particulares, así como las estrategias para promover la producción en cada uno de los estados de México. En el caso de Hidalgo, que se ubica en la región 18, los objetivos están orientados al fomento de la producción de piloncillo, renovación de los cañaverales y adopción de medidas de inocuidad.

La presencia de ingenios azucareros en México ha permitido la transformación, procesamiento y refinación del cultivo, potenciando las capacidades productivas en los principales estados productores (Veracruz, Oaxaca, Tabasco, Campeche). Aunque Veracruz es el mayor productor de caña de azúcar en el país, existen otros estados que tiene un mayor rendimiento gracias a su infraestructura en el sector, tal es el caso de Morelos. El estado de Hidalgo no cuenta con algún ingenio azucarero, de ahí que su aportación a la producción cañera del país es de menos del $1 \%$. Por lo anterior, la agroindustria en la entidad tiene grandes desafíos. Identificamos las siguientes áreas de oportunidad en el sector: a) fijar lineamientos de política agrícola que promuevan la producción cañera con el objetivo de orientar su diversificación hacia la producción de otros subproductos derivados de este cultivo, b) especializarse en la producción de piloncillo, pero con medidas de inocuidad y certificación orgánica que incrementen su valor en el mercado, c) acercar la producción campesina de Hidalgo con los ingenios azucareros de los estados colindantes con la finalidad de elaborar otros subproductos, como el azúcar refinada y d) incentivar la generación de energía alterna (etanol) a partir de la caña de azúcar.

\section{Referencias}

[1] Francis, M. B. (2000). Globalización, crisis auzcarera y luchas cañeras en los años noventa. Sociológica, 15 (44), 41-65.

[2] FIRA. (2016). Panorama Agroalimentario. México DF: FIRA.

[3] Aguilar, R. N., Galindo, M. G., Fortanelli, M. J., \& Contreras, S. C. (2011). Factores de competitividad de la agroindustria de la caña de azúcar en México. Región y Sociedad,23(52): Hermosillo, 261-297. 
[4] Wilkinson, J., \& Rocha, R. (2013). Tendencias de las agroindustrias, patrones e impactos en el desarrollo. En FAO, Agroindustrias para el desarrollo (págs. 51-102). Roma.

[5] De Janvry, A. (2013). Anexo: Agricultura para el desarrollo: implicaciones para las agroindustrias. En FAO, Agroindustrias para el desarrollo (págs. 285-306). Roma.

[6] Domínguez Ruvalcaba, L. (2005). Desarrollo regional y competitividad: la agroindustria azucarera en México. Nóesis. Revista de Ciencias Sociales y Humanidades, 15(27), 227-250.

[7] Gómez-Merino, F., \& et. al. (Noviembre de 2017). La diversificación de la agroindustria azucarera como estrategia para México. Agroproductividad, 10(11), 7-12.

[8] Santillán-Fernández, A., \& et. al. (2014). Dynamics of sugar cane production in México: 2000-2011. Agroproductividad, 7(6), 23-29.

[9] Diario Oficial de la Federación. (2015). Programa Institucional de Desarrollo del Comité Nacional para el Desarrollo Sustentable de la caña de azúcar 2015-2018., (pág. Primera Sección). México, D.F.

[10] León-Martínez, T., \& et. al. (2013). Paja de la caña de azúcar. Sus usos en la actualidad. (I. C. Azúcar, Ed.) ICIDCA. Sobre los Derivados de la Caña de Azúcar, 47(2), 13-22.

[11] Aguilar-Rivera, N. (2012). Paradigma de la diversificación de la agroindustria azucarera de México. (UAEM, Ed.) Convergencia. Revista de Ciencias Sociales, mayo-agosto (59), 187-213.

[12] Santillán-Fernández, A., \& et. al. (2014). Dynamics of sugar cane production in México: 2000-2011. Agroproductividad, 7(6), 23-29.

Referencias complementarias

[13] Cervantes, R. A. (s.f.). Inforural. Recuperado el 15 de Mayo de 2018, de Agrocluster, una alternativa para el campo: https://www.inforural.com.mx/agrocluster-una-alternativa-para-elcampo/

[14] CONADESUCA. (s.f.). El CICTCAÑA, Herramienta necesaria para el fortalecimiento de la investigación integral de la caña de azúcar en México. Recuperado el 31 de Mayo de 2018, de Comisión Nacional del Desarrollo Sustentable de la Caña de Azúcar: http://www.cndsca.gob.mx/Julio\%202010/Presentacion_Mesa_Trabajo _Tabasco_EL_CICTCANA.pdf

[15] CONADESUCA. (s.f.). Guia practica de los programas de apoyo de la SAGARPA para productores de caña de azúcar. Recuperado el 20 de Mayo de 2018, de Comité Nacional para el Desarrollo Sustentable de la Caña de Azúcar: http://www.sagarpa.gob.mx/quienesomos/datosabiertos/conadesuca/Pa ginas/default.aspx

[16] CONEVAL. (2018). Consejo Nacional de Evaluación de la Política de Desarrollo Social. Recuperado el 16 de Mayo de 2018, de https://www.coneval.org.mx/Medicion/IRS/Paginas/Que-es-el-indicede-rezago-social.aspx

[17] Desconocido. (s.f.). La caña de azúcar en México. Recuperado el 22 de Mayo de 2018, de Azúcar que endulca mi vida: http://siaprendes.siap.gob.mx/contenidos/3/03-cana-azucar/contexto3.html

[18] DOF. (s.f.). Programa Nacional de la Agroindustria de la caña de $\begin{array}{lccc}\text { azúcar } & 2014-2018 . & \text { Obtenido } & \text { de } \\ \text { http://dof.gob.mx/nota_detalle.php?codigo }=5343244 \& \text { fecha }=02 / 05 / 20\end{array}$ http://dof.gob.mx/nota_detalle.php? codigo $=5343244 \&$ fecha $=02 / 05 / 20$
14

[19] FAO. (2013). Agroindustrias para el desarrollo. Roma.

[20] FIRA. (s.f.). Panorama Agroalimentario, Azúcar 2015. Recuperado el 11 de Junio de 2018, de Fideicomisos Instituidos en Relación con la Agricultura:

https://www.gob.mx/cms/uploads/attachment/file/61947/Panorama_Ag roalimentario_Az_car_2015.pdf

[21] INEGI. (2010). Censo de Población y Vivienda 2010. Recuperado el 16 de Mayo de 2018, de http://www.beta.inegi.org.mx/proyectos/ccpv/2010/
[22] SAGARPA. (s.f.). Acciones y Programas, Programa de Fomento a la Agricultura. Recuperado el 26 de Mayo de 2018, de Secretaría de Agricultura, Ganadería, Desarrollo Rural, Pesca y Alimentación: http://www.sagarpa.gob.mx/agricultura/Programas/proagro/Paginas/Ob jetivo.aspx

[23] SAGARPA. (s.f.). Atlas Agroalimentario 2017. Recuperado el 06 de Junio de 2018, de Secretaría de Agricultura, Ganadería, Desarrollo Rural, Pesa y Alimentación: http://online.pubhtml5.com/clsi/ibhs/

[24] SAGARPA. (s.f.). Caña de Azúcar Mexicana. Recuperado el 30 de Abril de 2018, de Planeación Agrícola Nacional 2017-2030: https://www.gob.mx/cms/uploads/attachment/file/256427/B_sicoCa_a_de_az_car.pdf

[25] SAGARPA. (s.f.). Caña de azúcar: cierra la cosecha 2017 con 56 millones de toneladas. Recuperado el 09 de Junio de 2018, de Secretaría de Agricultura, Gandería, Desarrollo Rural, Pesca y Ganadería: https://www.gob.mx/siap/articulos/cana-de-azucar-cierra-la-cosecha2017-con-56-millones-de-toneladas?idiom=es

[26] SAGARPA. (s.f.). Datos Abierto, CONADESUCA. Recuperado el 31 de Mayo de 2018, de Secretaría de Agricultura, Ganadería, Desarrollo Rural, Pesa y Alimentación: http://www.sagarpa.gob.mx/quienesomos/datosabiertos/conadesuca/Pa ginas/default.aspx

[27] SAGARPA. (s.f.). Importancia de la Agroindustria de la Caña de Azúcar. Recuperado el 14 de Mayo de 2018, de Secretaría de Agricultura, Ganadería, Desarrollo Rural, Pesca y Alimentación: http://www.sagarpa.gob.mx/agricultura/Documents/Cultivos\%20Agroi ndustriales/Impactos\%20Caña.pdf

[28] SAGARPA. (s.f.). Ingenio Azucarero, el primer paso de la transformación del azúcar. Recuperado el 19 de Mayo de 2018, de Secretaría de Agricultura, Ganadería, Desarrollo Rural, Pesca y Alimentación:

http://www.sagarpa.gob.mx/Delegaciones/veracruz/boletines/Paginas/2 016B067.aspx\#

[29] SAGARPA. (s.f.). Los 57 ingenios de la agroindustria de la caña de azúcar y sus respectivos abastecedores de Caña. Recuperado el 01 de Junio de 2018, de Secretaría de Agricultura, Ganadería, Desarrollo Rural, Pesca y Alimentación: http://www.sagarpa.gob.mx/Transparencia/Pot\%202013/CONADESU CA/Padron\%20Ingenios\%202012.pdf

[30] SAGARPA. (s.f.). Programas de Agricultura, Proagro. Recuperado el 19 de Mayo de 2018, de Secretaría de Agricultura, Ganadería, Desarrollo Rural, Pesca y Alimentación: http://www.sagarpa.gob.mx/agricultura/Programas/proagro/Paginas/Ob jetivo.aspx 